\title{
Criminologie
}

\section{La restriction de l'usage du tabac en prison au Québec : effets sur le climat carcéral}

\author{
Serge Brochu, Lyne Chayer, Benoit Lasnier, Michael Cantinotti, Louise Guyon \\ et Ann Royer
}

Volume 43, numéro 2, automne 2010

Les 50 ans de l'École de criminologie : aperçu de la recherche d'ici et d'ailleurs

URI : https://id.erudit.org/iderudit/1001772ar

DOI : https://doi.org/10.7202/1001772ar

Aller au sommaire du numéro

Éditeur(s)

Les Presses de l’Université de Montréal

ISSN

0316-0041 (imprimé)

1492-1367 (numérique)

Découvrir la revue

Citer cet article

Brochu, S., Chayer, L., Lasnier, B., Cantinotti, M., Guyon, L. \& Royer, A. (2010). La restriction de l'usage du tabac en prison au Québec : effets sur le climat carcéral. Criminologie, 43(2), 127-151. https://doi.org/10.7202/1001772ar

\section{Résumé de l'article}

L'objectif principal de cet article est de mieux connaître les perceptions de personnes détenues en ce qui a trait aux effets sur le climat carcéral d'un règlement qui vise à restreindre l'usage du tabac dans les établissements de détention québécois. Une méthodologie mixte a été employée afin de recueillir des données auprès de 113 personnes détenues dans trois établissements de détention du Québec. Les données quantitatives ont été analysées de manière descriptive et comparative, alors que les données qualitatives ont fait l'objet d'une analyse thématique. Les résultats de l'étude démontrent que les personnes détenues, en désaccord pour la majorité avec l'implantation du nouveau règlement, ont perçu que son implantation a contribué à une hausse des tensions, du trafic de cigarettes et de leur valeur sur le marché noir. Selon leur position ou leur pouvoir, cette situation peut s'avérer profitable pour certains détenus alors qu'elle accroît la vulnérabilité d'autres détenus. L'hypothèse de départ voulant que l'entrée en vigueur d'un nouveau règlement sur le tabagisme entraîne des perturbations du climat carcéral est soutenue par les résultats obtenus.
Ce document est protégé par la loi sur le droit d'auteur. L'utilisation des services d'Érudit (y compris la reproduction) est assujettie à sa politique d'utilisation que vous pouvez consulter en ligne.

https://apropos.erudit.org/fr/usagers/politique-dutilisation/ 


\title{
La restriction de l'usage du tabac en prison au Québec: effets sur le climat carcéral
}

\author{
Serge Brochu \\ Professeur titulaire \\ École de criminologie, Université de Montréal \\ serge.brochu@umontreal.ca
}

\section{Lyne Chayer}

Agente de planification, de programmation et de recherche Institut national de santé publique du Québec (INSPQ) lyne.chayer@inspq.qc.ca

\section{Benoit Lasnier}

Agent de planification, de programmation et de recherche Institut national de santé publique du Québec (INSPQ)

benoit.lasnier@inspq.qc.ca

\section{Michael Cantinotti ${ }^{1}$}

Agent de planification, de programmation et de recherche Institut national de santé publique du Québec (INSPQ)

michael.cantinotti@inspq.qc.ca

\section{Louise Guyon}

Consultante

Institut national de santé publique du Québec (INSPQ)

louise.guyon@inspq.qc.ca

\author{
Ann Royer \\ Responsable de l'évaluation \\ Équipe habitudes de vie/maladies chroniques DRSP de la Capitale-Nationale \\ ann.royer@ssss.gouv.qc.ca
}

1. Michael Cantinotti était boursier postdoctoral du Programme stratégique de formation en recherche en santé publique et en santé des populations des IRSC et du RRSPQ (Université McGill et Direction de santé publique de la Capitale-Nationale) au cours de l'étude. 
RÉSUMÉ - L'objectif principal de cet article est de mieux connaître les perceptions de personnes détenues en ce qui a trait aux effets sur le climat carcéral d'un règlement qui vise à restreindre l'usage du tabac dans les établissements de détention québécois. Une méthodologie mixte a été employée afin de recueillir des données auprès de 113 personnes détenues dans trois établissements de détention du Québec. Les données quantitatives ont été analysées de manière descriptive et comparative, alors que les données qualitatives ont fait l'objet d'une analyse thématique. Les résultats de l'étude démontrent que les personnes détenues, en désaccord pour la majorité avec l'implantation du nouveau règlement, ont perçu que son implantation a contribué à une hausse des tensions, du trafic de cigarettes et de leur valeur sur le marché noir. Selon leur position ou leur pouvoir, cette situation peut s'avérer profitable pour certains détenus alors qu'elle accroît la vulnérabilité d'autres détenus. L'hypothèse de départ voulant que l'entrée en vigueur d'un nouveau règlement sur le tabagisme entraîne des perturbations du climat carcéral est soutenue par les résultats obtenus.

MOTS-CLÉS - Établissements de détention, règlement sur le tabac, climat carcéral.

\section{Introduction ${ }^{2}$}

Chaque année, des dizaines de milliers de décès attribuables au tabagisme sont enregistrés au Canada, ce qui place les maladies liées au tabagisme parmi les principales causes de mortalité évitables au pays (Makomaski et al., 2004). Lorsqu'on le compare aux autres produits psychoactifs, le tabac est celui qui est le plus problématique d'un point de vue sanitaire (Rehm et al., 2006). Le tabagisme ne nuit pas uniquement à la santé des fumeurs, mais également à celle des non-fumeurs car la fumée du tabac dans l'environnement (FTE) expose les individus à un mélange de fumée exhalée par les fumeurs et de fumée provenant de la combustion des produits du tabac. Ces deux types de fumée contiennent plus de 250 substances toxiques ou cancérigènes (U.S. Department of Health and Human Services, 2005 ; Guérin et al., 2005).

\section{La consommation de tabac en milieu carcéral}

Plusieurs études démontrent que la proportion de fumeurs s'avère beaucoup plus élevée parmi la population carcérale que parmi la population générale. Les taux de prévalence recensés au sein de la population

2. La réalisation de la présente recherche a été rendue possible grâce à la contribution financière du ministère de la Santé et des Services sociaux et à une subvention de recherche provenant de l'Interdisciplinary Capacity Enhancement (ICE) Program (Small Project Funding) pour la partie qualitative de l'évaluation. Les opinions exprimées dans ce document ne reflètent pas nécessairement celles du ministère de la Santé et des Services sociaux et du ministère de la Sécurité publique du Québec. 
carcérale sont de l'ordre de $72 \%$ au Canada (Robinson et Mirabelli, 1996) et de $80 \%$ aux États-Unis (Cropsey et al., 2004). À titre indicatif, $19 \%$ des Canadiens âgés de 15 ans et plus avaient été identifiés comme des fumeurs en 2007 (Santé Canada, 2007). Un bon nombre d'études mentionnent également la forte présence de la FTE dans les prisons (Linhorst et al., 2001; Hammond et Emmons, 2005). Le chef du Service fédéral de la santé publique des États-Unis (Surgeon General) rapportait en 2006 que les prisons comptaient parmi les lieux habités enregistrant les plus fortes concentrations de FTE (U.S. Department of Health and Human Services, 2006).

\section{Les règlements sur le tabagisme en milieu carcéral}

Face à cette situation, l'Organisation mondiale de la santé a publié en 2007 un rapport concernant les meilleures pratiques sanitaires en prison qui recommande l'adoption de mesures face au tabagisme et à la FTE. Dans le domaine de la santé publique, l'une des mesures considérées les plus efficaces pour préserver la santé de l'ensemble des détenus et $\mathrm{du}$ personnel est l'adoption de règlements qui interdisent l'usage du tabac dans les prisons (World Health Organization - Regional Office for Europe, 2007). Parmi les principales raisons mentionnées pour soutenir cette mesure, on note l'atteinte à la santé des non-fumeurs causée par la FTE et la nécessité d'instaurer en détention des standards égaux à ceux observés dans la société, telle l'interdiction de fumer dans les lieux publics, en raison du risque excessif pour la santé que représente l'exposition à la FTE (Wilcox, 2007).

Au cours des dix dernières années, l'usage du tabac dans les établissements correctionnels a donc progressivement été remis en question, tant aux États-Unis qu'au Canada. À la fin de 2006, 58\% des États américains interdisaient complètement l'usage de la cigarette dans leurs établissements de détention (Wilcox, 2007). Au Canada, des mesures ont été mises en place dès l'année 2000, notamment en Saskatchewan et en Nouvelle-Écosse. En 2008, 11 des 13 provinces et territoires avaient complètement interdit la consommation de tabac dans leurs prisons (Royer et Cantinotti, 2008). Le Québec est la dernière province canadienne à mettre en place un règlement interdisant la consommation de tabac à l'intérieur et à l'extérieur de tous ses centres de détention. Ce règlement est entré en vigueur le 5 février 2008, soit 10 ans après 
l'interdiction de fumer dans les lieux de travail ${ }^{3}$. Toutefois, trois jours après son introduction, le 8 février 2008, ce règlement a été amendé pour permettre aux détenus de fumer dans les cours extérieures des prisons «afin que l'application de l'interdiction de fumer se fasse le plus facilement possible pour les usagers du tabac» (ministère de la Sécurité publique, 8 février 2008).

\section{Les effets d'un règlement sur le tabagisme en milieu carcéral}

Diminution de la FTE. Une étude récemment réalisée en Caroline du Nord afin d'évaluer les effets d'un règlement sur le tabagisme à l'intérieur des établissements de détention rapporte qu'en moyenne, les niveaux de particules de fumée secondaire du tabac respirables en suspension dans l'air ont diminué de $77 \%$ dans les prisons à la suite de l'application de la loi (Proescholdbell et al., 2008). Ce résultat amène les auteurs à suggérer que les lois qui interdisent l'usage du tabac dans les établissements de détention peuvent réduire de manière significative l'exposition à la FTE chez les détenus, les visiteurs et les membres du personnel. Elles pourraient également contribuer à réduire la consommation de tabac des individus qui vivent en milieu carcéral (Proescholdbell et al., 2008). Cropsey et Kristeller (2005) nuancent ce dernier point en avançant que ce sont les fumeurs légers qui auraient davantage tendance à restreindre leur consommation de tabac. Les fumeurs qui poursuivent leur consommation de tabac souffriraient généralement d'une dépendance plus sévère à la nicotine (Sieminska et al., 2006).

Augmentation de la contrebande et du trafic de tabac. Certains administrateurs redoutent une augmentation du trafic de tabac dans les prisons à la suite de la mise en place d'un règlement qui interdit son usage. Dans

3. Au Québec, le gouvernement a mis en place différentes mesures législatives qui restreignent l'usage, la vente et la promotion des produits du tabac. En 1998 entrait en vigueur l'interdiction de fumer dans les lieux de travail (Gouvernement du Québec, 2008). Par la suite, la révision de la Loi sur le tabac adoptée en 2005 élargissait cette interdiction à tout le territoire québécois, incluant les lieux publics (les restaurants, les bars, les hôtels, les salles de bingo, etc.), de même que certains établissements publics (les établissements de santé et de services sociaux, les cégeps, les universités, etc.). Les administrateurs de certains milieux tels que les centres d'hébergement, les hôpitaux de soins de longue durée, les centres d'hébergement psychiatrique et les centres de détention ont toutefois la possibilité de se soustraire à cette loi. Pour plus de renseignements à ce sujet, voir les Bulletins d'information publiés sur le site www.msss.gouv.qc.ca. 
ce contexte, certains détenus se sont effectivement retrouvés impliqués dans le trafic de cigarettes ou d'autres infractions reliées au tabac (Linhorst et al., 2001). Lankenau (2001) indique que l'architecture complexe des établissements de détention, les déplacements des détenus à l'intérieur et à l'extérieur, l'implication du personnel dans la contrebande de tabac et la plus ou moins grande vigilance des agents correctionnels quant à l'application de la loi peuvent contribuer à l'apparition d'un trafic illégal de tabac dans les centres de détention. Cet auteur suggère également que l'interdiction de fumer dans les prisons tend à modifier le rôle généralement joué par la cigarette: habituellement utilisée comme monnaie d'échange, elle devient alors un objet de contrebande. Ce phénomène est également rapporté par Kauffman et al. (2008) qui indiquent que 17 des 49 administrations correctionnelles étasuniennes incluses dans leur étude déclaraient avoir remarqué une recrudescence de la contrebande de tabac à la suite du resserrement des politiques sur le tabagisme au sein des établissements de détention. Par ailleurs, Chavez et al. (2004) notent qu'une conséquence potentielle de cette situation est que le tabac supplante les drogues illégales comme substance de contrebande de prédilection en prison. À ce sujet, le Service correctionnel du Canada (SCC, 24 août 2006) rapporte que le tabac est effectivement devenu l'objet interdit le plus populaire depuis l'entrée en vigueur d'une interdiction de fumer dans les lieux internes des pénitenciers fédéraux.

Hausse des actes violents. La crainte d'assister à une augmentation du niveau de violence entre les détenus, ainsi qu'entre ces derniers et les membres du personnel, constitue l'une des principales préoccupations des administrateurs de prisons par rapport aux politiques anti-tabac (Vaughn et Del Carmen, 1993). Toutefois, une étude de Patrick et Marsh (2001) réalisée auprès de 51 administrations correctionnelles étasuniennes, dont 7 appliquaient une interdiction complète de fumer et 44 une interdiction limitée, ne valide pas ces craintes. Dans les faits, une augmentation de la violence n'a été rapportée que dans deux de ces institutions, alors qu'une recrudescence des tensions entre les détenus n'a été signalée que par $20 \%$ des prisons à la suite des nouvelles mesures. Une étude plus récente menée par Kauffman et al. (2008) confirme les précédents résultats. Celle-ci rapporte qu'aucun événement violent majeur n'a été répertorié dans l'ensemble des institutions de détention étatsuniennes à la suite de l'entrée en vigueur de politiques plus strictes 
en matière de tabagisme. Divers auteurs mentionnent toutefois que l'interdiction de l'usage du tabac a été associée dans certains cas à un accroissement de problèmes déjà présents dans le milieu, notamment l'intimidation et la prostitution (Vaughn et Del Carmen, 1993; Lankenau, 2001; Butler et al., 2007). Somme toute, en reprenant la distinction proposée par Chauvenet (2006), il est possible de croire que la violence liée aux contentieux interpersonnels demeure inchangée alors que certaines violences inhérentes au cadre institutionnel de la prison se trouvent exacerbées par les réglementations anti-tabac.

Au Canada, le SCC a interdit en 2006 l'usage du tabac dans les lieux internes de ses établissements, tout en continuant d'en tolérer l'usage dans les cours extérieures (Service correctionnel du Canada: 12 juillet 2005, 27 juin 2007). Selon le SCC, les craintes émises lors de l'annonce de ce nouveau règlement quant à l'augmentation de la violence n'étaient pas fondées (Service correctionnel du Canada: 24 août 2006).

Dans cette optique, il est apparu indispensable de mieux connaître les perceptions de personnes détenues quant aux effets de ces règlements sur le climat carcéral ${ }^{4}$. L'hypothèse de l'étude en question veut que l'entrée en vigueur d'un nouveau règlement sur le tabagisme entraîne des perturbations du climat carcéral, tant sur le plan relationnel (e.g. intimidation, violence...) qu'en fonction de l'apparition de comportements interdits (non-respect des lois anti-tabac, trafic...). Il est à noter qu'au Québec, aucune étude n'avait à ce jour été menée sur les effets d'un règlement qui vise à restreindre l'usage du tabac dans les établissements de détention.

\section{Méthode}

\section{Milieux étudiés}

Trois établissements de détention ont été retenus afin de constituer le milieu d'étude: deux dans la région de Montréal (Établissement de détention de Montréal [Bordeaux] et Maison Tanguay), et un troisième

4. L'étude dont proviennent les résultats présentés ici comportait quatre objectifs, les effets perçus de l'interdiction partielle du tabagisme sur le climat carcéral étant l'un d'entre eux. Les autres objectifs visaient à décrire: 1) les habitudes associées à l'usage du tabac chez les personnes détenues, leurs intentions et motivations à cesser de fumer, ainsi que les besoins exprimés à ce sujet; 2) les effets du règlement qui interdit de fumer à l'intérieur des établissements de détention sur la consommation de tabac des personnes détenues et sur leur exposition à la FTE; et 3) la façon dont les employés intègrent le changement de règlement sur le tabac dans leurs pratiques professionnelles. 
dans la région de Québec (Établissement de détention de Québec). Il s'agit d'établissements provinciaux qui accueillent des personnes condamnées à des peines maximales de deux ans moins un jour d'emprisonnement, ainsi que des prévenus en attente de procès et des détenus en attente de transfert. L'Établissement de détention de Montréal (Bordeaux) est une institution masculine pouvant accueillir un très grand nombre de détenus, alors que la Maison Tanguay est un établissement de détention pour les femmes aux dimensions beaucoup plus modestes. L'Établissement de détention de Québec constitue, selon les standards québécois, une institution de taille moyenne qui comprend à la fois des ailes de détention masculines et féminines.

Des personnes-ressources ont été désignées pour chacun des établissements inclus dans l'enquête afin de faciliter la coordination de l'étude avec l'équipe de recherche. Des informations recueillies auprès de ces personnes ont permis de constater certaines différences entre les trois établissements de détention quant à leur réglementation interne (tableau 1). Bien que l'achat de produits du tabac soit réglementé dans chacun des établissements, les quantités permises varient d'un endroit à l'autre. Ainsi, dans les établissements de détention qui accueillent des détenus de sexe masculin, on permet la vente de quantités de tabac plus importantes que dans les centres de détention pour femmes. En fait, dans un des établissements étudiés, les détenues n'ont droit qu'à une allocation de cinq cigarettes par jour. Par ailleurs, les détenus ne peuvent fumer que lors de leur sortie quotidienne d'une heure dans la cour extérieure, sauf dans l'un des centres où deux sorties par jour sont accordées.

\section{Procédure de sélection des répondants}

Le recrutement des participants a été mené selon une procédure d'autosélection, c'est-à-dire sous forme d'une participation volontaire. Il ne s'agissait pas nécessairement d'obtenir un échantillon représentatif de la population carcérale; cependant, des efforts ont été faits afin d'obtenir une certaine représentativité en recrutant davantage d'hommes que de femmes de même qu'un nombre plus important de personnes fumeuses que non fumeuses. Les personnes détenues ont reçu une invitation à participer à l'étude par des affiches exposées dans les différents secteurs des prisons. Ainsi, les personnes qui pouvaient être mal à l'aise à l'idée de participer à la recherche ne subissaient aucune pression de la part 
TA B LE A U 1

Description de la réglementation interne selon les établissements

\begin{tabular}{|l|l|l|l|}
\hline Établissements & $\begin{array}{l}\text { Quantité de tabac } \\
\text { permise }\end{array}$ & $\begin{array}{l}\text { Nombre de } \\
\text { sorties dans la } \\
\text { cour extérieure }\end{array}$ & $\begin{array}{l}\text { Briquets/ } \\
\text { allumettes }\end{array}$ \\
\hline $\begin{array}{l}\text { Établissement } \\
\text { de détention } \\
\text { de Québec } \\
\text { (section hommes) }\end{array}$ & $\begin{array}{l}\text { Cantine: } 3 \text { paquets } \\
\text { de cigarettes, ou } \\
50 \text { grammes de tabac } \\
\text { en sac }+1 \text { paquet de } \\
\text { cigarettes par semaine }\end{array}$ & $\begin{array}{l}\text { Une fois par jour } \\
\text { (1 heure) }\end{array}$ & $\begin{array}{l}\text { Disponibles } \\
\text { à la cantine }\end{array}$ \\
\hline $\begin{array}{l}\text { Établissement } \\
\text { de détention } \\
\text { de Québec } \\
\text { (section femmes) }\end{array}$ & $\begin{array}{l}\text { Projet pilote } \\
\text { implanté en mai } \\
2008: \text { remise de } 5 \\
\text { cigarettes par jour }\end{array}$ & $\begin{array}{l}\text { Une fois par jour } \\
\text { (1 heure) }\end{array}$ & $\begin{array}{l}\text { Disponibles } \\
\text { à la cantine }\end{array}$ \\
\hline $\begin{array}{l}\text { Établissement } \\
\text { de détention } \\
\text { de Montréal } \\
\text { (Bordeaux) }\end{array}$ & $\begin{array}{l}\text { Cantine: } 3 \text { paquets } \\
\text { ou } 80 \text { grammes de } \\
\text { tabac en sac par } \\
\text { semaine }\end{array}$ & $\begin{array}{l}\text { Une fois par jour } \\
\text { (1 heure) }\end{array}$ & $\begin{array}{l}\text { Les briquets } \\
\text { sont interdits. } \\
\text { Les allumettes } \\
\text { sont remises } \\
\text { avec l'achat de } \\
\text { cigarettes à la } \\
\text { cantine } \\
\text { (5 pochettes/ } \\
\text { sem.) }\end{array}$ \\
\hline Maison Tanguay & $\begin{array}{l}\text { Cantine: } 2 \text { paquets } \\
\text { de cigarettes ou } \\
50 \text { grammes de tabac } \\
\text { en sac par semaine }\end{array}$ & $\begin{array}{l}\text { Une ou deux fois } \\
\text { par jour selon } \\
\text { leur pause de } \\
\text { travail sur } \\
\text { semaine et deux } \\
\text { fois par jour la } \\
\text { fin de semaine } \\
(1 \text { heure) }\end{array}$ & $\begin{array}{l}\text { Les briquets et } \\
\text { les allumettes } \\
\text { sont interdits. } \\
\text { Lors des sorties } \\
\text { dans la cour, les } \\
\text { agents prêtent } \\
\text { un briquet aux } \\
\text { fumeuses et le } \\
\text { récupèrent } \\
\text { ensuite }\end{array}$ \\
\hline
\end{tabular}

1. Avant la promulgation du règlement, l'achat de cigarettes n'était pas limité. Il y avait toutefois un plafond quant au montant autorisé pour des achats de tabac hebdomadaires à la cantine. Peu après le changement de règlement, la possession de tabac à l'intérieur a été interdite. Des cartes échangeables contre des cigarettes sont vendues aux détenues, ces dernières reçoivent alors leurs cigarettes au moment de la sortie extérieure.

des chercheurs. Quatre intervieweuses ont reçu une formation de la part des chercheurs et ont effectué les rencontres avec les détenus. Les détenus participants répondaient en premier lieu au questionnaire quantitatif $^{5}$, et un certain nombre d'entre eux étaient ensuite invités à

5. Les questions étaient posées par les intervieweuses, qui transcrivaient ensuite la réponse du participant sur une version papier du questionnaire. 
participer à l'entrevue qualitative semi-dirigée ${ }^{6}$. Toutes les rencontres ont eu lieu dans un local fermé prévu à cet effet, afin d'assurer la confidentialité des propos. La collecte des données s'est déroulée au cours des mois d'août à octobre 2008.

\section{Instruments}

Afin de répondre aux objectifs de l'étude, une méthode mixte a été privilégiée, combinant les approches quantitative et qualitative (Tashakkori et Teddlie, 1998). Avec l'objectif d'assurer la prise en compte des dimensions objectives et subjectives du sujet d'étude dans une perspective complémentaire et constructiviste (Karsenti et SavoieZajc, 2000; Pinard et Potvin, 2004), un questionnaire combiné avec une entrevue semi-dirigée ont été utilisés. Le questionnaire aborde, entre autres, les perceptions des détenus quant aux effets du nouveau règlement sur l'environnement et le climat carcéral, en lien avec l'approvisionnement en produits du tabac et quant à l'application du règlement. Pour les entrevues qualitatives, un guide d'entrevue semi-structurée a été élaboré. Les personnes rencontrées ont été invitées à décrire comment elles percevaient le nouveau règlement anti-tabac en milieu carcéral. Les thèmes suivants ont été abordés dans l'entrevue: les perceptions quant au nouveau règlement, le climat carcéral à la suite de l'implantation du règlement, l'approvisionnement en tabac et l'adaptation des fumeurs au nouveau règlement.

\section{Participants}

Le tableau 2 décrit les 113 participants au volet quantitatif de l'étude. Tous établissements confondus, la majorité des répondants (69\%) étaient des détenus, $26 \%$ étaient des prévenus et $5 \%$ avaient un autre statut. Au moment de l'entrevue, $45 \%$ des répondants étaient détenus depuis moins de 101 jours, $18 \%$ entre 101 et 200 jours, $27 \%$ entre 201 et 400 jours, et $10 \%$ depuis plus de 400 jours. L'échantillon des répondants est composé majoritairement de détenus de sexe masculin (65\%). Un répondant sur quatre (25\%) avait entre 18 et 30 ans, $29 \%$ entre 31 et 40 ans, $30 \%$ entre 41 et 50 ans et $16 \%$ plus de 51 ans. La majorité

6. Les questions étaient posées par les intervieweuses; le contenu de l'entrevue était enregistré sur un support audio. 
TABLEAU 2

Description sociodémographique de l'échantillon selon les établissements

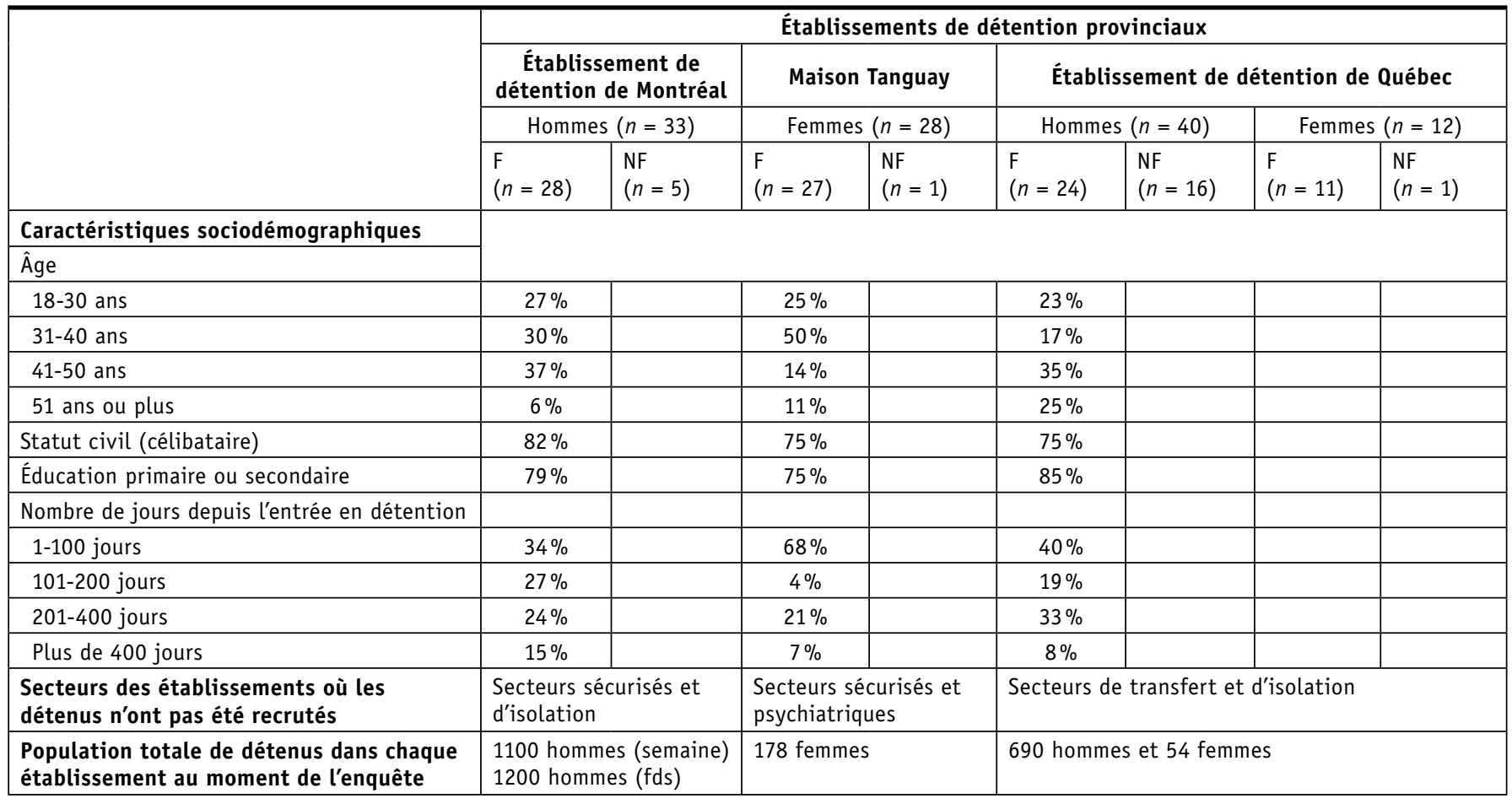

Note: $\mathrm{F}=$ fumeurs; $\mathrm{NF}=$ non-fumeurs. 
(77\%) n'était pas en couple et avait une éducation de niveau primaire ou secondaire $(81 \%)$. Les répondants $(80 \%)$ étaient des usagers de tabac, dont $94 \%$ fumaient sur une base quotidienne. Il est à noter que les femmes se retrouvaient en plus grande proportion que les hommes à se considérer comme des personnes fumeuses $(95 \% \text { versus } 71 \%)^{7}$.

Les 45 répondants retenus pour le volet qualitatif de l'étude présentent des caractéristiques similaires à l'ensemble de l'échantillon utilisé dans le volet quantitatif. Effectivement, le sous-échantillon qualitatif se compose d'une majorité d'hommes $(\mathrm{n}=27)$, d'individus qui n'étaient pas en couple $(n=32)$ et de personnes ayant complété une éducation de niveau primaire ou secondaire $(n=38)$. Des effectifs identiques $(n=13)$ de répondants inclus dans le sous-échantillon qualitatif étaient respectivement âgés de 18 à 30 ans, de 31 à 40 ans ou de 41 à 50 ans, alors qu'un plus petit effectif de répondants était âgé de plus de 51 ans $(n=6)$. Parmi les 45 répondants, 35 étaient des fumeurs, une très large majorité d'entre eux se déclarant fumeurs quotidiens $(n=34)$. Les proportions calculées à partir de ces effectifs sont très similaires à celles répertoriées parmi l'ensemble de l'échantillon de recherche. Encore ici, les femmes $(n=18)$ se retrouvent en plus grande proportion que les hommes $(\mathrm{n}=27)$ à se considérer comme des personnes fumeuses (89\% contre $70 \%)$.

\section{Traitement et analyse des données}

Les réponses des participants au questionnaire ont été saisies et analysées de manière descriptive et comparative à l'aide du logiciel SPSS version 16.0. Les intervalles de confiance à $95 \%$ ont été calculés à partir de la méthode exacte de Clopper-Pearson.

Les entrevues qualitatives ont été enregistrées sur un support audio et retranscrites sous forme de verbatim. Une analyse thématique des entrevues a été effectuée selon six grandes catégories inspirées des objectifs de la recherche. Les sous-catégories ont pour leur part émergé des données brutes obtenues, processus s'apparentant à une approche générale d'analyse inductive (Blais et Martineau, 2006). Cette analyse thématique a été exécutée à l'aide du logiciel NVivo 7. Le matériel tiré de l'analyse qualitative a été utilisé pour approfondir et nuancer certains résultats provenant des analyses quantitatives.

7. $\chi^{2}=9,00, p<0,01$. 
Par ailleurs, bien que le cadre d'analyse privilégié ait été fortement centré sur les objectifs de la recherche et ait laissé place au matériel émergeant, des éléments provenant de la sociologie du milieu carcéral se sont également avérés utiles pour permettre une meilleure compréhension des résultats obtenus. Ces éléments concernent essentiellement la vision de la prison comme une société qui possède une culture ou une sous-culture propre (Combessie, 2004). À cet égard, notons le concept de prisoniérisation, qui constitue une forme d'assimilation au milieu carcéral faisant en sorte que les personnes incarcérées adoptent de nouvelles habitudes de vie, que ce soit en termes d'habillement, de déplacements, de langage ou d'habitudes alimentaires (Vacheret et Lemire, 2007). Vacheret et Lemire (2007) précisent toutefois qu'en raison des changements survenus dans le milieu carcéral depuis les années 1940, époque au cours de laquelle le concept de prisoniérisation a été proposé pour la première fois, plusieurs facteurs s'y rattachant ont eu tendance à s'atténuer au fil du temps. Bien que le phénomène de la prisoniérisation puisse être moins marqué qu'il ne l'était auparavant, il n'en demeure pas moins que la concentration de centaines ou de milliers de personnes dans un espace restreint faisant l'objet de contrôles serrés peut provoquer des phénomènes propres à une sous-culture (Vacheret et Lemire, 2007). Cette sous-culture se caractérise tant par un code de valeurs ${ }^{8}$ partagé par les détenus que par la solidarité et la cohésion qui se manifestent entre eux. Bien que ce cadre d'analyse faisant référence à une sous-culture et au phénomène de prisoniérisation ait été initialement privilégié, d'autres aspects provenant de la sociologie des prisons permettent de faciliter la compréhension des résultats recueillis. En effet, le manque de tabac crée des tensions entre détenus, ce qui met à l'épreuve l'hypothèse d'une solidarité entre détenus. À cet égard, la description des établissements de détention contemporains offerte par Chantraine (2006), qui utilise la terminologie post-disciplinaire afin de décrire la réalité actuelle des prisons, permet d'appréhender cette dimension. L'auteur soutient que le «système bonbon ${ }^{9}$ » et l'instrumentalisation intensifiée des rapports sociaux en détention ont remplacé la

8. Ce code, ni officiel ni écrit, soutient des principes tels que la loyauté entre les détenus, garder son sang-froid, ne pas s'exploiter entre détenus, faire preuve de courage et ne jamais faiblir devant l'adversité, ne pas frayer avec l'ennemi (les gardiens et la direction) (Vacheret et Lemire, 2007).

9. Le «système bonbon» est un terme québécois pour caractériser le système de privilèges individuels et collectifs en prison (Chantraine, 2006). 
force et les mesures disciplinaires dans la production des types de comportements conformes aux objectifs de l'institution. Afin de minimiser le désordre, l'administration pénitentiaire et ses agents gèrent de façon pragmatique la vie quotidienne en prison en négociant, réprimant, privilégiant, instrumentalisant, opprimant, sanctionnant et récompensant les détenus (Chantraine, 2006). Ce type de gestion, qui fonctionne davantage aux récompenses qu'aux peines, se base sur l'octroi d'un confort relatif plutôt que sur des privations strictes et a pour conséquences, toujours selon Chantraine (2006), d'individualiser les stratégies d'adaptation, de fragmenter les espoirs individuels et d'intensifier l'instrumentalisation des rapports sociaux entre détenus et surveillants.

\section{Confidentialité et éthique}

Avant la collecte des données, les objectifs et les impacts possibles de la recherche étaient expliqués aux participants, qui devaient ensuite indiquer leur consentement libre et éclairé en signant un formulaire à cet effet. Toutes les mesures nécessaires ont été prises pour assurer l'anonymat des participants et la confidentialité des données. Le projet a reçu l'approbation d'un comité d'éthique.

\section{Résultats}

Les résultats seront présentés en deux temps. En premier lieu, la partie quantitative présentera les opinions et les perceptions brutes des détenus rencontrés. Ensuite, dans la partie qualitative, ces opinions seront approfondies à partir des entrevues menées auprès du sous-échantillon.

\section{Aspects saillants qui émergent des questionnaires}

La présentation des résultats quantitatifs fera l'objet de deux sections distinctes. La première section traite de l'opinion des détenus par rapport au règlement ainsi que des effets de ce dernier sur la vie carcérale, plus précisément sur le plan du climat en prison et du trafic ${ }^{10}$ de cigarettes. Par la suite, la deuxième section aborde la question des métho-

10. Le terme «trafic» fait ici référence à l'action d'effectuer, de manière illicite, des transactions sur des produits du tabac à l'intérieur de l'établissement de détention, que ces produits aient été acquis de manière légale ou non au départ. 
des d'approvisionnement en cigarettes et du prix des cigarettes achetées sur le marché noir ${ }^{11}$.

La plupart des répondants fumeurs $(86 \%)$ se sont déclarés en désaccord avec le règlement interdisant l'usage du tabac à l'intérieur des établissements de détention. Du côté des non-fumeurs, ce sont $44 \%$ des répondants qui affirment être en désaccord avec l'interdiction de fumer à l'intérieur des établissements de détention. Cet écart significatif $^{12}$ entre l'opinion des fumeurs et celle des non-fumeurs pourrait avoir eu des répercussions sur climat carcéral, la majorité (85\%) des détenus interrogés ayant indiqué que les tensions entre détenus s'étaient aggravées depuis l'entrée en vigueur du nouveau règlement (tableau 3). Autant les répondants fumeurs que les non-fumeurs ont fait cette observation ${ }^{13}$.

Une tendance similaire émerge au sujet des tensions entre détenus et membres du personnel. En effet, une majorité de répondants (76\%) pense qu'elles se sont aggravées, quoique cette perception soit plus prononcée chez les fumeurs $(80 \%)$ que chez les non-fumeurs $(59 \%)^{14}$. Interrogés sur la présence de tensions entre les membres du personnel, un peu plus de la moitié des détenus (53\%) a indiqué avoir remarqué une augmentation des tensions depuis la promulgation du règlement le 8 février 2008. Les fumeurs et les non-fumeurs ont une opinion similaire à ce sujet ${ }^{15}$.

Par ailleurs, qu'ils soient fumeurs ou non-fumeurs ${ }^{16}$, la majorité des détenus (69\%) croit que le nombre d'événements violents a augmenté dans l'établissement carcéral depuis que le règlement est entré en vigueur. Ce constat semble également s'appliquer pour ce qui a trait à la perception du nombre de plaintes formulées par les détenus ${ }^{17}$. Indépendamment de leur statut tabagique (fumeur ou non-fumeur) ${ }^{18}$, une majorité des répondants $(64 \%)$ perçoit que le nombre de plaintes a augmenté à la suite de l'entrée en vigueur du nouveau règlement.

11. Le terme «marché noir» fait ici référence au système de revente illégale des produits du tabac établi à l'intérieur de l'établissement de détention.

12. Test exact de Fisher, $\mathrm{p}<0,01$.

13. Test exact de Fisher, $\mathrm{p}=, 05$.

14. $\chi^{2}=3,90, p<0,05$.

15. $\chi^{2}=0,05, p=0,82$.

$16 \chi^{2}=0,01, p=0,94$.

17. Toutes les plaintes faites par les détenus sont considérées, et non pas seulement celles concernant le tabac.

18. $\chi^{2}=1,17, p=0,28$. 
Parallèlement à ces constats sur le climat en détention, le phénomène du trafic illicite de cigarettes en milieu carcéral paraît avoir été affecté par la mise en place du règlement sur le tabagisme. En effet, la majorité des détenus, tant fumeurs (61\%) que non-fumeurs (59\%), indique que le trafic de cigarettes a augmenté depuis le 8 février 2008 (tableau 3) ${ }^{19}$.

TAB LEA U 3

Impacts perçus du règlement sur la vie carcérale par les personnes détenues

\begin{tabular}{|l|c|c|c|c|c|c|}
\hline \multicolumn{2}{|c|}{ Augmentation } & \multicolumn{2}{l|}{ Pas de changement } & \multicolumn{2}{c|}{ Diminution } \\
\cline { 2 - 7 } & Fumeurs & $\begin{array}{c}\text { Non- } \\
\text { fumeurs }\end{array}$ & Fumeurs & $\begin{array}{c}\text { Non- } \\
\text { fumeurs }\end{array}$ & Fumeurs & $\begin{array}{c}\text { Non- } \\
\text { fumeurs }\end{array}$ \\
\hline $\begin{array}{l}\text { Tensions entre } \\
\text { les détenus } \\
\text { (N=112) }\end{array}$ & $88 \%$ & $70 \%$ & $12 \%$ & $30 \%$ & $0 \%$ & $0 \%$ \\
\hline $\begin{array}{l}\text { Tensions entre } \\
\text { les détenus et } \\
\text { les membres du } \\
\text { personnel (N = 105) }\end{array}$ & $80 \%$ & $59 \%$ & $19 \%$ & $32 \%$ & $1 \%$ & $9 \%$ \\
\hline $\begin{array}{l}\text { Tensions entre } \\
\text { les membres du } \\
\text { personnel (N=53) }\end{array}$ & $54 \%$ & $50 \%$ & $44 \%$ & $42 \%$ & $2 \%$ & $8 \%$ \\
\hline $\begin{array}{l}\text { Événements } \\
\text { violents dans } \\
\text { la prison } \\
\text { (N=101) }\end{array}$ & $69 \%$ & $70 \%$ & $28 \%$ & $25 \%$ & $3 \%$ & $5 \%$ \\
\hline $\begin{array}{l}\text { Plaintes formulées } \\
\text { par les détenus } \\
\text { (N = 87) }\end{array}$ & $66 \%$ & $53 \%$ & $34 \%$ & $37 \%$ & $0 \%$ & $11 \%$ \\
\hline $\begin{array}{l}\text { Trafic de cigarettes } \\
\text { (N = 109) }\end{array}$ & $61 \%$ & $59 \%$ & $28 \%$ & $27 \%$ & $12 \%$ & $14 \%$ \\
\hline
\end{tabular}

Il a été mentionné précédemment que la majorité des détenus interrogés avaient signalé une augmentation des tensions entre détenus après la promulgation du règlement; il reste cependant à déterminer si celui-ci a amené des répercussions sur la fréquence d'occurrence de conflits entre fumeurs et non-fumeurs, telle qu'elle est perçue par les répondants. À ce sujet, il est possible de noter que la perception des détenus fumeurs ne se révèle pas différente de celle des non-fumeurs ${ }^{20}$. Plus précisément,

19. $\chi^{2}=0,03, p=0,88$.

20. $\chi^{2}=0,90, p=0,34$. 
$37 \%$ des fumeurs et $48 \%$ des non-fumeurs ont déclaré avoir observé souvent ou occasionnellement des conflits entre fumeurs et nonfumeurs.

Les répercussions engendrées par le nouveau règlement sur les méthodes d'approvisionnement en cigarettes ont également été examinées (tableau 4). Sachant que l'achat à la cantine représente le seul moyen légal de se procurer des produits du tabac en milieu correctionnel, il n'est pas surprenant de constater que $92 \%$ des détenus fumeurs ont rapporté utiliser cette méthode pour s'en procurer. Néanmoins, une proportion non négligeable de fumeurs a rapporté avoir acheté des cigarettes sur le marché noir (28\%), avoir obtenu des cigarettes par le biais d'échanges (28\%), avoir gagné des cigarettes au jeu $(13 \%)$ ou avoir reçu des cigarettes gratuitement $(17 \%)$.

TA B LE A U 4

Méthodes d'approvisionnement en cigarettes

\begin{tabular}{|l|c|c|}
\hline \multirow{2}{*}{} & \multicolumn{2}{|c|}{$\begin{array}{c}\text { Répondants qui rapportent utiliser cette méthode } \\
\text { d'approvisionnement en cigarettes }\end{array}$} \\
\cline { 2 - 3 } & Proportion (\%) & Intervalles de confiance (95\%) \\
\hline Cantine & $92 \%$ & $85 \%-97 \%$ \\
\hline Marché noir & $28 \%$ & $19 \%-38 \%$ \\
\hline Échange de biens/services & $28 \%$ & $19 \%-38 \%$ \\
\hline Gain au jeu & $13 \%$ & $7 \%-22 \%$ \\
\hline Don & $17 \%$ & $10 \%-26 \%$ \\
\hline
\end{tabular}

Univers: répondants qui sont fumeurs quotidiens ou occasionnels $(N=90)$.

Les détenus ont également été interrogés au sujet du prix des cigarettes sur le marché noir à l'intérieur de l'établissement de détention. La majorité des détenus, qu'ils soient fumeurs $(72 \%)$ ou non-fumeurs (67\%), rapportent que le prix des cigarettes sur le marché noir a augmenté à la suite de l'entrée en vigueur du règlement. Le paquet, qui se vendait au prix moyen de $11 \$$, serait passé à 18 \$ après la promulgation du règlement.

\section{Aspects saillants qui émergent des entrevues qualitatives}

Plusieurs détenus associent les tensions perçues aux restrictions concernant la quantité de tabac qu'il est permis d'acheter et aux occasions où 
il est possible de fumer. Ces restrictions occasionnent un manque de nicotine chez les fumeurs, manque qui peut se traduire par des tensions et de l'agressivité:

Bien, tout le monde est plus stressé, tout le monde est plus sur les dents, tout le monde est plus agressif, c'est sûr là. Tu sais, des personnes qui sont habituées de fumer deux, trois paquets de cigarettes par jour, là, ils se limitent à une cigarette, deux cigarettes par jour c'est comme... Eh! C'est comme un sevrage assez brutal. (Marie-Claude, détenue, fumeuse)

Les effets associés au manque de nicotine sont perçus par les détenus interrogés comme étant importants pour les gros fumeurs et les personnes incarcérées depuis une longue période. Ces fumeurs avaient depuis longtemps l'habitude de fumer aux moments et selon les quantités qu'ils désiraient. Des détenus soulignent également que le manque de nicotine s'ajoute parfois aux sevrages à l'alcool et aux drogues. L'un d'eux rapporte croire que les agents correctionnels fumeurs, qui doivent eux aussi se soumettre au règlement, peuvent également avoir des sensations de manque qui se traduisent par davantage d'agressivité.

Plusieurs détenus rapportent que les tensions sont plus importantes la journée précédant la cantine lorsque les détenus ont épuisé leur réserve de produits du tabac:

Bon bien le mardi, c'est le gros party, tout le monde est heureux, y ont leur cantine, le tabac est là, on s'aime, on est tous des frères. Mais quand la fin de semaine arrive, puis qu'il te reste un fond de blague ${ }^{21}$, t'es de mauvaise humeur. T'as le goût de casser la gueule à tout le monde. C'est pas vivable là, ça s'engueule tout le temps. (Hugo, détenu, non-fumeur)

Confrontés au manque de cigarettes, certains détenus n'hésiteraient pas à voler leurs compagnons de cellule:

À toutes les semaines là, quelques jours avant la cantine, bien, ceux qui manquent de tabac sont plus agressifs, c'est évident, là on le voit, ça, puis moi j'ai vu des batailles, j'ai vu ensuite des punitions parce que... des gens qui ont été envoyés au trou pour avoir volé une cigarette ou deux cigarettes, c'est un peu ridicule là, mais c'est ça. (Jean-Pierre, détenu, fumeur)

Les non-fumeurs ne sont pas épargnés durant cette période, d'une part parce que le climat est tendu et d'autre part parce qu'ils sont sollicités

21. Une blague de tabac représente du tabac à rouler vendu en sac; les quantités de tabac vendues dans ces sacs se retrouvent dans le tableau 1 qui présente la description des milieux étudiés. 
d'acheter du tabac pour les fumeurs. De plus, parce qu'ils sont peu nombreux, ils s'exposent à des menaces s'ils se plaignent du non-respect $\mathrm{du}$ règlement. Un non-fumeur exprime d'ailleurs vivre un sentiment d'isolement, de mépris et subir des menaces:

J'me sens tout seul face à ça étant donné le pourcentage élevé de fumeurs. Euh, c'est rare même que je vois des non-fumeurs qui peuvent se tenir. [...] Fait que je trouve beaucoup que c'est de la tolérance que j'travaille, pis je sais que ça règlera pas mon sort, d'avoir recours à la violence, mais souvent j'accumule, je le dis, ça change pas. [...] En tout cas, j'me sens ben gros méprisé pis euh... rabaissé. On m’a fait des menaces, lorsque j'ai fait des mémos pis des feuilles de plaintes, euh... [...] Des menaces physiques euh... verbales euh... de la part de codétenus. (Nicolas, détenu, nonfumeur)

Plusieurs participants expliquent qu'à la base, avant même de considérer les effets du règlement sur le tabagisme, l'incarcération est en elle-même lourde de conséquences et source de tensions. Selon ces participants, l'interdiction de fumer ne fait qu'ajouter aux tensions vécues par les détenus:

Bien un stress! Dans le sens que les filles rentrent ici, ils [elles] sont en attente de sentence, ils [elles] savent pas pour combien de temps qu'ils [qu'elles] vont pogner [...], leurs enfants sont à l'extérieur, y [elles] sont pas là pour veiller à qu'est-ce qu'ils [elles] veillent d'habitude pour leurs enfants. C'est ça. D’apprendre à ta famille que t'es en détention; c'est toutes des affaires que c'est stressant déjà là. Tu sais c'est..., tu fais déjà un sevrage de toutes ces affaires-là que t'es privée, que t'es contrôlée même sur qu'est-ce que tu manges, bien là, en plus, il faut que t'arrêtes de fumer, c'est comme... C'est beaucoup. C'est beaucoup. C'est lourd. (Pascale, détenue, fumeuse)

Quelques participants vont au-delà de la description du climat de tensions et de pressions et rapportent la survenue d'événements violents qu'ils attribuent au marché noir qui s'est créé autour des cigarettes. Selon eux, l'incapacité de payer des dettes accumulées pour l'achat de cigarettes sur le marché noir, le fait de ne pas remettre un emprunt pour des produits du tabac ou de refuser de prêter ou de donner des cigarettes sont susceptibles de provoquer des événements violents. Des détenus ayant vécu d'autres expériences d'incarcération estiment que de tels événements ne se produisaient pas auparavant en lien avec le tabac. 
Certains détenus affirment que le règlement leur permet de s'enrichir en faisant le commerce de cigarettes sur le marché noir. D’autres détenus, fumeurs et non-fumeurs, rapportent des désagréments reliés au marché noir. Ce dernier aurait augmenté en raison des nouvelles restrictions sur le tabagisme en milieu carcéral, et en particulier en raison des limites imposées aux quantités de tabac vendues en cantine. Ces participants croient que le marché noir des cigarettes, où les prix sont revus à la hausse, est préjudiciable pour les détenus qui n'ont pas les ressources financières suffisantes pour s'en procurer. Ces derniers peuvent aller jusqu'à se priver de nourriture ou se prostituer afin d'obtenir des produits du tabac:

Y'a des filles qui vont s'empêcher de manger pis y vont vendre leur repas, leur dessert, pour des cigarettes. (Carole, détenue, non fumeuse)

J'en ai vu moi qui se prostituaient pour un paquet là. [...] Aie, regarde un moment donné, quand t'es rendu là là... (Pierre-Luc, détenu, fumeur)

Ainsi, il semble que pour certains détenus, le marché noir peut être profitable, alors qu'il exacerbe la situation de vulnérabilité d'autres détenus. Une participante estime d'ailleurs que peu de détenus possèdent un important pouvoir d'achat à l'intérieur des murs:

[Il n'] y en pas beaucoup là qui ont les moyens d'acheter ce qu'ils veulent, quand ils veulent puis comme ils veulent. (Geneviève, détenue, fumeuse)

Bien que le discours des personnes interviewées démontre que l'échange de biens contre des cigarettes est un phénomène qui était déjà présent dans les établissements de détention, il semble que les nouvelles restrictions sur le tabac ont exacerbé la situation. Les participants ont mentionné que les biens échangés pour obtenir des cigarettes étaient des bijoux, des vêtements, des médicaments et des drogues illicites.

\section{Discussion}

L'hypothèse de départ voulant que l'entrée en vigueur d'un nouveau règlement sur le tabagisme entraîne des perturbations du climat carcéral est confirmée par les résultats obtenus. Cette perturbation semble liée à la rareté récente d'un produit d'usage courant (rappelons que la grande majorité des détenus fumaient avant leur admission en détention) et l'augmentation du prix du tabac qui s'ensuit. Le fait que le prix des produits interdits en détention augmente en fonction de la difficulté 
à s'en procurer avait déjà été établi en ce qui concerne les drogues illicites (Plourde et Brochu, 2002); la présente étude apporte un élément nouveau en mettant en évidence que cette augmentation de prix cible un produit tout à fait légal, à la suite de la mise en place d'un règlement qui en restreint la vente et l'usage. Le prix du tabac sur le marché noir est instable, évoluant en fonction de la rareté, au fur et à mesure que le stock hebdomadaire s'épuise. Le climat carcéral paraît suivre une tendance parallèle. Ainsi, le règlement a contribué à accentuer le rôle des cigarettes comme objet de valeur au sein des établissements de détention. Comme l'argent circule peu dans les prisons, le nouveau rapport de force créé par la valeur accrue des cigarettes donne lieu à un grand nombre de tractations entre détenus; pour satisfaire leur besoin et payer leurs dettes, certains vont alors échanger des biens essentiels tels leurs médicaments ou accepter de rendre des services, dont certains sont d'ordre sexuel. Nulle surprise alors de constater que la majorité des détenus fumeurs se sont dits en désaccord avec ce règlement.

Les activités de troc des produits du tabac provoquent l'exploitation des plus faibles et des conflits entre les plus forts, tout en contribuant à «enrichir» certains non-fumeurs et trafiquants de cigarettes qui voient là une occasion d'affaires inédite. Ce résultat rejoint ceux de Kauffman et al. (2008) et Lankenau (2001), qui signalaient que la cigarette perdait sa valeur de monnaie d'échange pour devenir un objet de contrebande en soi à la suite de l'interdiction de fumer dans des établissements carcéraux.

Toutefois, bien que les tentatives d'approvisionnement en produits du tabac aient modifié les rapports entre les détenus et contribué à l'exploitation des plus faibles, il ne semble pas que cela se traduise par une augmentation significative d'activités délictueuses comme le vol ou le recours à la violence entre les personnes détenues. Cette situation, qui peut à première vue sembler étrange dans un contexte carcéral, pourrait s'expliquer d'une part par les caractéristiques des personnes détenues dans les prisons provinciales, et d'autre part par ce qui est désigné dans la littérature scientifique sur le milieu carcéral comme étant le «code des détenus». En effet, le code d'honneur des détenus veut que ceux-ci ne se volent pas entre eux (Vacheret et Lemire, 2007). De plus, il faut savoir que plusieurs personnes détenues dans des établissements provinciaux le sont pour des délits de faible gravité, comme le non-paiement d'amendes. Cette observation confirme les résultats de plusieurs études réalisées dans d'autres pays (Patrick et Marsh, 2001; Kauffman et al., 2008). 
Bien qu'ils confirment l'hypothèse du code de valeurs et de la sousculture à l'égard des vols et de la violence entre détenus, les résultats obtenus font cependant apparaître une tension quant à l'effet de la structure sur les liens de solidarité entre détenus. D’un côté, la domination procure une unité entre les détenus (hypothèse de la sousculture), de l'autre, elle individualise les stratégies d'adaptation, elle «divise». La sensation de «manque» de nicotine, qui est précisément un ressenti individuel, quoique concernant plusieurs des détenus et résultant d'un effet de structure, influence les rapports entre détenus et entre détenus et surveillants. Ces dimensions «individualisantes» de la régulation des comportements en détention ont d'ailleurs été mentionnées par Chantraine (2006).

\section{Conclusion}

Cette étude montre clairement que certains effets pervers ont été observés à la suite de la mise en place du règlement anti-tabac dans les prisons provinciales du Québec. Ces effets doivent être mis dans le contexte d'une restriction de l'offre en tabac et des opportunités de fumer dans un milieu où la majorité de la clientèle est constituée de fumeurs. Ces effets sont de deux types. D'une part, il y aurait la recrudescence du marché noir des cigarettes qui impliquerait à la fois des fumeurs et des non-fumeurs, et d'autre part, le développement par certains détenus de moyens alternatifs qui leur permettent de continuer à fumer à l'intérieur des établissements de détention. Ces effets de la restriction de l'offre en tabac ne peuvent par ailleurs être dissociés des autres privations et frustrations extrêmes de la vie en prison: privation de liberté, de biens et de services, de relations hétérosexuelles, d'autonomie et de sécurité (Sykes, 1958).

Cette recherche met l'accent sur plusieurs questions qui vont au-delà de la simple observation de l'apparition d'effets pervers à la suite de l'application d'un règlement qui restreint l'usage du tabac en détention. Si le choix d'instaurer un tel règlement se justifie par le devoir de tout établissement d'assurer la protection de la santé des personnes qui y sont incarcérées ainsi que du personnel qui y travaille, sa mise en œuvre suscite également des questions qui dépassent le cadre de la santé publique.

La Loi sur le tabac exempte sous certaines conditions les milieux de vie de l'application de restrictions sur le tabagisme (Gouvernement du 
Québec, 2008, 2009)22. Ainsi dans les centres d'hébergement et des soins de longue durée, l'établissement peut autoriser un résident à fumer dans sa chambre et peut aménager un fumoir. Cependant, l'établissement doit s'assurer que cela n'enfreint pas les règles de sécurité et n'affecte pas la santé des travailleurs et autres résidents du centre. Il est ainsi nécessaire de se demander si les établissements de détention doivent être assimilés ou non à des milieux de vie (en particulier les cellules). La réponse à cette question, qui n'est ni d'ordre sanitaire ni criminologique, peut avoir des répercussions pratico-légales importantes.

Par ailleurs, cette étude peut également étayer certains débats théoriques sur la sociologie carcérale. En outre, des questionnements sont mis en évidence quant au degré de spécificité de l'institution carcérale. À un extrême, elle pourrait être appréhendée comme une institution à part, alors qu'à l'autre extrême, elle serait plutôt perçue comme une institution «comme les autres» (Chantraine, 2000). De plus, les conséquences observées à la suite de l'interdiction et des restrictions sur les denrées rares telles que le tabac, auxquelles s'ajoutent un ensemble d'autres privations (Sykes, 1958), mettent en relief la violence - manifeste ou latente - des institutions carcérales (Chauvenet, 2006).

\section{Références}

Blais, M., \& Martineau, S. (2006). L'analyse inductive générale: description d'une démarche visant à donner un sens à des données brutes. Analyses qualitatives, 26 (2), 1-18.

Butler, T., Richmond, R., Belcher, J., Wilhelm, K., \& Wodak, A. (2007). Should smoking be banned in prisons? Tobacco Control, 16 (5), 291-293.

Chantraine, G. (2000). La sociologie carcérale: approches et débats théoriques en France. Déviance et société, 24 (3), 297-381.

Chantraine, G. (2006). La prison post-disciplinaire. Déviance et Société, 30 (3), 273-288.

Chauvenet, A. (2006). Privation de liberté et violence: le despotisme ordinaire en prison. Déviance et société, 30 (2), 373-388.

Chavez, R. S., Oto-Kent, D. S., Porter, J., Brown, K., Quirk, L., \& Lewis, S. (2004). Tobacco policy, cessation, and education in correctional facilities. West Sacramento, CA: Health Education Council. Consulté le 5 février 2010 sur www.healthedcouncil.org.

22. www2.publicationsduquebec.gouv.qc.ca/dynamicSearch/telecharge.php? type=2\&file=/T_0_01/T0_01.htm. 
Combessie, P. (2004). Intégration sociale des anciens détenus. Analyse des logiques de la justice pénale et de leurs effets. In J. Poupart (Ed.), Au-delà du système pénal: l'intégration sociale et professionnelle de groupes judiciarisés et marginalisés (231-248). Québec: Les Presses de 1'Université du Québec.

Cropsey, K. L., Eldridge, G. D., \& Ladner, T. (2004). Smoking among female prisoners: An ignored public health epidemic. Addictive Behaviors, 29 (2), 425-431.

Cropsey, K. L., \& Kristeller, J. L. (2005). The effects of a prison smoking ban on smoking behavior and withdrawal symptoms. Addictive Behaviors, 30 (3), 589-594.

Guérin, D., Guyon, L., Fournier, M., Gillet, M., Payette, Y., \& Laguë, J. (2005). La fumée de tabac secondaire. Effets sur la santé et politiques de contrôle de l'usage du tabac dans les lieux publics. Québec: Institut national de santé publique du Québec.

Hammond, S. K., \& Emmons, K. M. (2005). Inmate exposure to secondhand smoke in correctional facilities and the impact of smoking restrictions. Journal of Exposure Science $\mathfrak{F}$ Environmental Epidemiology, 15 (3), 205-211.

Karsenti, T., \& Savoie-Zajc, L. (2000). Introduction à la recherche en évaluation. Université de Sherbrooke: Éditions CRP.

Kauffman, R. M., Ferketich, A. K., \& Wewers, M. E. (2008). Tobacco policy in American prisons, 2007. Tobacco Control, 17 (5), 357-360.

Lankenau, S. E. (2001). Smoke'em if you got'em: Cigarette black markets in U.S prisons and jails. The Prison Journal, 81 (2), 142-161.

Linhorst, D. M., Knight, K., Johnston, J. S., \& Trickey, M. (2001). Situational influences on the implementation of a prison-based therapeutic community. The Prison Journal, 81 (4), 436-453.

Gouvernement du Québec (2008). Loi sur le tabac (à jour au 1er juin 2008), L.R.Q. chapitre T0.01 C.F.R. Consulté le 3 mars 2009 sur: www.publicationsduquebec.gouv.qc.ca.

Gouvernement du Québec (2009). L.R.Q. chapitre S-40.1. Loi sur le système correctionnel du Québec. Consulté le 5 février 2010 sur: www.msp.gouv.qc.ca.

Makomaski Illing, E. M., \& Kaiserman, M. J. (2004). Mortality attributable to tobacco use in Canada and its regions. Canadian Journal of Public Health, 95 (1), 38-44.

Ministère de la Sécurité publique (8 février 2008). Interdiction de fumer dans les établissements de détention du Québec: application de mesures d'assouplissement. Consulté le 6 juin 2009 sur: www.msp.gouv.qc.ca.

Patrick, S., \& Marsh, R. (2001). Current tobacco policies in U.S. adult male prisons. The Social Science Journal, 38 (1), 27-37.

Pinard, R., \& Potvin, P. (2004). Le choix d'une approche méthodologique mixte en éducation. Recherches qualitatives, 24, 58-80.

Plourde, C., \& Brochu, S. (2002). Drugs in prison: A break in the pathway. Substance Use \& Misuse, 37 (1), 47-63. 
Proescholdbell, S. K., Foley, K. L., Johnson, J., \& Malek, S. H. (2008). Indoor air quality in prisons before and after implementation of a smoking ban law. Tobacco Control, 17 (2), 123-127.

Rehm, J., Taylor, B., Patra, J., \& Gmel, G. (2006). Avoidable burden of disease : Conceptual and methodological issues in substance abuse epidemiology. International Journal of Methods in Psychiatric Research, 15 (4), 191-111.

Robinson, D., \& Mirabelli, L. (1996). Résumé des constatations du Sondage national auprès des détenus réalisé en 1995 par le SCC. Ottawa: Service correctionnel du Canada. Consulté le 5 janvier 2010 sur: www.csc-scc.gc.ca/text/rsrch/ briefs/b14/b14e-fra.shtml.

Royer, A., \& Cantinotti, M. (2008). Évaluation de la formation en cessation tabagique offerte aux agents de soins de santé des établissements de détention de Québec, Rivière-des-Prairies et de Montréal (Bordeaux). Québec: Direction de santé publique de la Capitale-Nationale.

Santé Canada (2007). Enquête de surveillance de l'usage du tabac au Canada (ESUTC). La prévalence du tabagisme 1999-2007. Consulté le 14 août 2009 sur: www.gosmokefree.ca

Service correctionnel du Canada (12 juillet 2005). Le SCC annonce un plan visant à réduire l'exposition à la fumée secondaire à l'intérieur de tous les établissements correctionnels fédéraux. Communiqués de presse - National. Consulté le 31 mars 2009 sur: www.csc.scc.gc.ca/text/media/ntl-fra.shtml

Service correctionnel du Canada (24 août 2006). Révision de la politique du Service correctionnel du Canada sur l'exposition à la fumée secondaire. Ottawa: SCC.

Service correctionnel du Canada (27 juin 2007). Mise en application prochaine d'une interdiction complète de fumer dans tous les établissements correctionnels fédéraux du Service correctionnel du Canada. Communiqués de presse - National. Consulté le 3 janvier 2010 sur www.csc-scc.gc.ca/text/media/ ntlrls/2007/07-06-27-fra.shtml.

Sieminska, A., Jassem, E., \& Konopa, K. (2006). Prisoners' attitudes towards cigarette smoking and smoking cessation: A questionnaire study in Poland. BMC Public Health, 6 (1), doi: 10.1186/1471-2458-6-181.

Sykes, G. M. (1958). The Society of Captives: A Study of a Maximum Security Prison. New Jersey: Princeton University Press.

Tashakkori, A., \& Teddlie, C. (1998). Mixed methodology: Combining qualitative and quantitative approaches. Thousand Oaks, CA: Sage.

U.S. Department of Health and Human Services (2005). 11th Report on Carcinogens. Consulté le 2 avril 2009 sur: www.ntp.niehs.nih.gov/ntp/roc/roc11.zip.

U.S. Department of Health and Human Services (2006). The bealth consequences of involuntary exposure to tobacco smoke. A report of the Surgeon General. Consulté le 15 juin 2008 sur www.surgeongeneral.gov/library/secondhandsmoke.

Vacheret, M., \& Lemire, G. (2007). Anatomie de la prison contemporaine. Montréal: Presses de l'Université de Montréal.

Vaughn, M. S., \& Del Carmen, R. V. (1993). Smoke-free prisons : Policy dilemmas and constitutional issues. Journal of Criminal Justice, 21 (2), 151-171. 
Wilcox, S. C. (2007). Secondhand smoke signals from prison. Michigan Law Review, 105 (8), 2081-2103.

World Health Organization - Regional Office for Europe (2007). Health in prisons - A WHO guide to the essentials in prison health. Copenhagen, Denmark: World Health Organization. Consulté le 23 juin 2008 sur: www.asca.net/ documents/e90174-HealthInPris.pdf.

\begin{abstract}
The aim of this paper is to gain perspective on the social climate found in Quebec's provincial correctional facilities since the introduction of an indoor smoking ban. Data was collected from 113 inmates incarcerated in three provincial correctional facilities, using a mixed methods design. Quantitative data were analyzed using descriptive and comparative statistics, while qualitative data were the object of a thematic analysis. Results from the study show that inmates, for the most part in disagreement with the new regulation banning indoor smoking, believe its implementation has played a role in increasing social tensions and cigarette trafficking, as well as contributing to a rise in the price of cigarettes on the black market. Depending on their position and influence within the prison environment, this situation was perceived to be either profitable or detrimental to the inmates. The initial hypothesis that the implementation of a new regulation on tobacco use would disrupt the prison's social climate is supported by the results obtained from this study.
\end{abstract}

KEYWORDS - Correctional facilities, anti-tobacco regulation, prison social climate, indoor smoking ban.

RESUMEN - El objetivo del presente artículo es conocer mejor las percepciones de los presos respecto de los efectos en el clima de la prisión generados por un reglamento orientado a restringir el uso del tabaco en los establecimientos de detención quebequenses. Se utilizó una metodología mixta para recabar datos de 113 detenidos en tres establecimientos de Quebec. Los datos cuantitativos se analizaron de modo descriptivo y comparativo mientras que los cualitativos fueron objeto de un análisis temático. Los resultados del estudio muestran que los detenidos, en desacuerdo mayoritario con la aplicación del nuevo reglamento, perciben que su implantación contribuyó a un aumento de las tensiones, el tráfico de cigarrillos y el precio de los mismos en el mercado negro. Esta situación puede resultar rentable para unos y en detrimento de otros, según su posición e influencia en la cárcel, con lo que se contribuye a incrementar la inequidad entre los presos. Los resultados obtenidos permitieron confirmar la hipótesis inicial, es decir que la entrada en vigor de un nuevo reglamento sobre el tabaquismo introduce perturbaciones en el clima penitenciario.

PALABRAS CLAVE - Establecimientos penitenciarios, reglamento sobre tabaco, clima penitenciario. 